Review began 12/28/2021 Review ended 01/09/2022 Published 01/26/2022

(c) Copyright 2022

Kharsa et al. This is an open access article distributed under the terms of the Creative Commons Attribution License CC-BY 4.0. which permits unrestricted use, distribution, and reproduction in any medium, provided the original author and source are credited.

\section{Constrictive Pericarditis: An Unusual Presentation of Rheumatoid Vasculitis}

\author{
Adnan Kharsa ${ }^{1}$, Medhat Chowdhury ${ }^{1}$, Bryan E-Xin Tan ${ }^{1}$, Mohammad Abu Sheikha ${ }^{2}$, Bipul Baibhav ${ }^{3}$ \\ 1. Internal Medicine, Rochester Regional Health, Rochester, USA 2. Cardiology, Rochester Regional Health, Rochester, \\ USA 3. Cardiology, Sands-Constellation Heart Institute, Rochester Regional Health, Rochester, USA
}

Corresponding author: Adnan Kharsa, adnan-kharsa@hotmail.com

\begin{abstract}
We describe a case of rheumatoid vasculitis with an atypical presentation of constrictive pericarditis. A 51year-old man who was previously admitted for diffuse lymphadenopathy, presented with chest pain and a lower extremity rash. Extensive workup including multimodality imaging, serology tests, and biopsy, resulted in the diagnosis of rheumatoid vasculitis.
\end{abstract}

Categories: Cardiology, Internal Medicine, Rheumatology

Keywords: diffuse lymphadenopathy, constrictive pericarditis, rheumatoid vasculitis, leukocytoclastic vasculitis, pericardiectomy

\section{Introduction}

Constrictive pericarditis (CP) is scarring of the pericardium that can occur due to various causes that include idiopathic $\mathrm{CP}$, viral illness, previous cardiac surgery, or less commonly connective tissue disorders $[1,2]$. Rheumatoid vasculitis (RV) is a serious extra-articular manifestation of rheumatoid arthritis (RA) in patients with a long-standing history of RA [3]. Involvement of cardiac tissue is present in $30 \%$ of patients with RV and this can manifest as pericarditis, myocarditis, coronary arteritis, or aortitis [3]. This article reports a patient who was initially found to have CP and later diagnosed with RV.

\section{Case Presentation}

A 51-year-old male presented to the emergency department with chest pain of four days duration. The pain was described as constant, non-radiating, and dull. The patient also reported a non-pruritic skin rash with similar onset, initially over the thighs, progressively extending to the lower abdomen. A review of systems disclosed progressive swelling of hands and legs. Physical examination was remarkable for jugular venous distension, bibasilar rales, and 2+ pitting edema of bilateral legs. No added sounds or friction rubs were noted. The abdomen was tender over the left lower quadrant with voluntary guarding. The patient had significant swelling and tenderness of bilateral hands. Violaceous rash extending from the thighs to the lower abdomen; slightly raised, non-blanching, and non-scaly.

A week before his presentation, he was admitted with non-radiating left-lower quadrant abdominal pain. Imaging including computed tomography (CT) scan of the chest and abdomen/ pelvis revealed diffuse lymphadenopathy involving the mediastinum, axillae, lower abdomen and pelvis, and a moderate-sized pericardial effusion. Echocardiography was remarkable for a dilated right atrium, dilated right ventricle with preserved systolic function, and a small circumferential pericardial effusion with mitral inflow respiratory variation without any evidence of tamponade physiology. Lymph node biopsies obtained from the right axilla and external iliac node subsequently were inconclusive.

\section{Past medical history}

Pertinent history was notable for bilateral carpal tunnel syndrome. He was recently evaluated for upper and lower extremities weakness and was diagnosed with sensorimotor polyneuropathy of indeterminate etiology. Pertinent family history disclosed a niece with juvenile arthritis. Social history disclosed a 15 -yearpack history of smoking.

\section{Differential diagnosis}

The differential diagnosis of skin rash, diffuse lymphadenopathy, and pericardial effusion is broad and includes lymphoma or malignancy, rheumatological disorder, vasculitis, cutaneous amyloidosis, cutaneous sarcoidosis, or infectious process.

\section{Further investigations}

Laboratory investigations were remarkable for mild normocytic anemia, elevated rheumatoid factor, and elevated anti-cyclic citrullinated peptide antibodies (Table 1). Electrocardiography (ECG) revealed 


\section{Cureus}

nonspecific T-wave changes with negative biomarkers. A coronary angiogram revealed normal coronary arteries. Cardiac MRI revealed thickened pericardium circumferentially with evidence of pericardial tethering, ventricular interdependence, and diffuse pericardial hyperenhancement suggesting pericardial inflammation (Figures $1 A-1 D$ ). A punch biopsy of the skin rash revealed dermal neutrophils, extravasation of red blood cells, and focal fibrinoid vascular change (Figures 2A, 2B). Findings were consistent with leukocytoclastic vasculitis.

\begin{tabular}{|c|c|c|}
\hline Blood test & Patient's lab value & Reference range \\
\hline $\mathrm{Hb}$ & $10 \mathrm{~g} / \mathrm{dL}$ & $13.0-18.0 \mathrm{~g} / \mathrm{dL}$ \\
\hline ESR & $74 \mathrm{~mm} / \mathrm{hr}$ & $0.0-23.0 \mathrm{~mm} / \mathrm{hr}$ \\
\hline CRP & 109 mg/L & $0.0-10.0 \mathrm{mg} / \mathrm{L}$ \\
\hline $\mathrm{RF}$ & $1700 \mathrm{IU} / \mathrm{mL}$ & $0.1-13.9 \mathrm{IU} / \mathrm{mL}$ \\
\hline Anti-CCP antibodies & $>300 \mathrm{U} / \mathrm{mL}$ & $<3 \mathrm{U} / \mathrm{mL}$ \\
\hline
\end{tabular}

\section{TABLE 1: Laboratory investigations.}

Hb: hemoglobin. ESR: sedimentation rate. CRP: c-reactive protein. RF: rheumatoid factor. Anti-CCP: anti-cyclic citrullinated peptide antibodies.
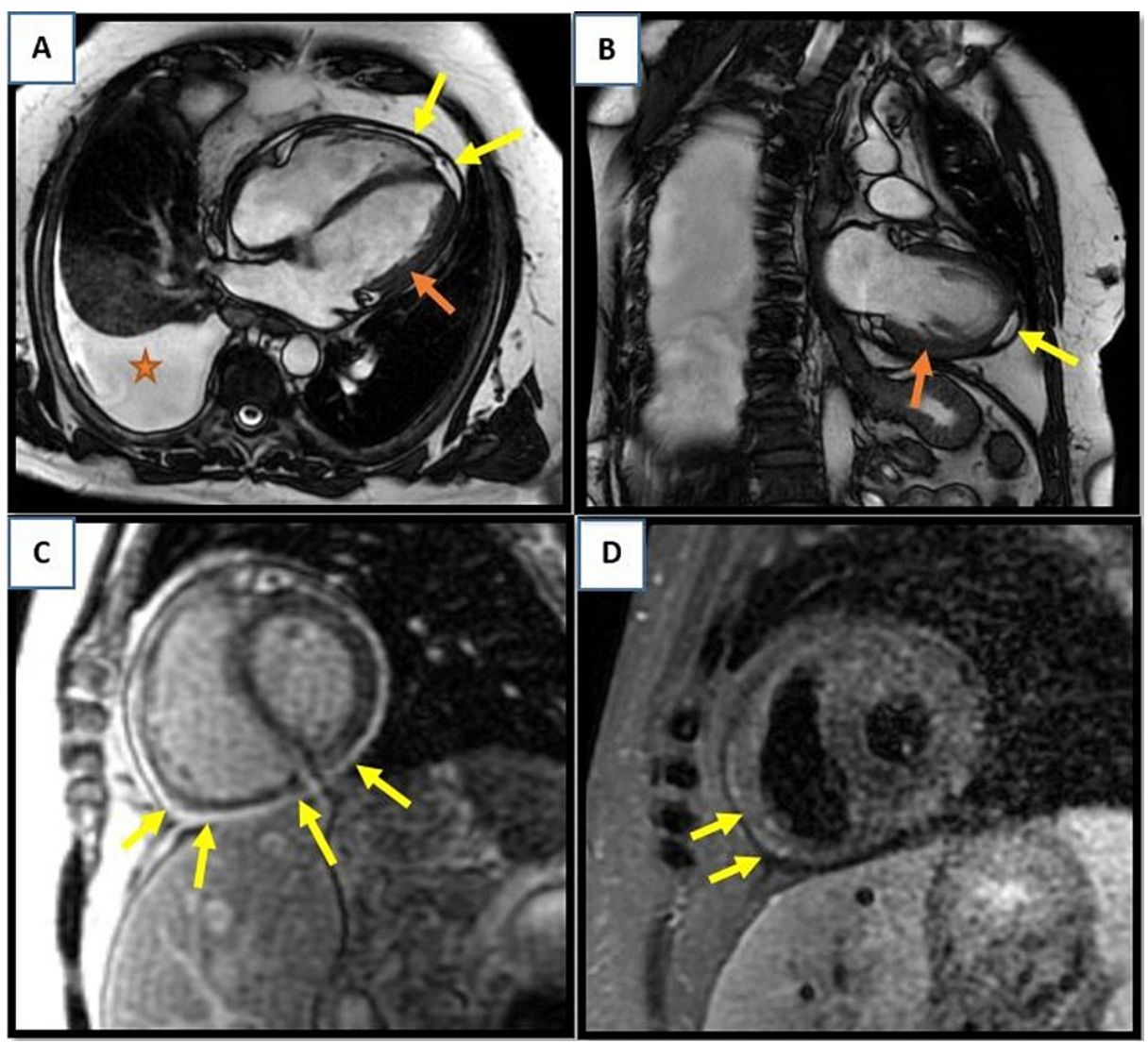

FIGURE 1: Cardiac MRI.

(A) Cross-sectional four-chamber and (B) coronal steady-state free precession (SSFP) images showing thickened pericardium (red arrows), small pericardial effusion (yellow arrows), and right-sided pleural effusion (red star). (C) Diffuse pericardial delayed enhancement is consistent with pericardial inflammation (arrows). (D) T2-weighted

image showing thickened pericardium (arrows). 


\section{Cureus}

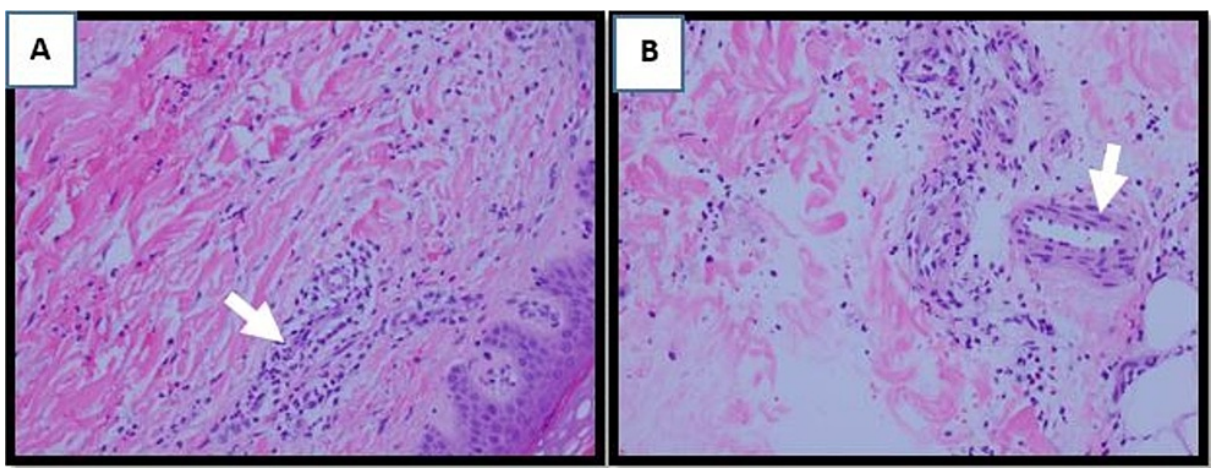

FIGURE 2: Skin biopsy results.

The skin biopsy reveals (A) neutrophilic infiltration (arrow) of the dermis and extravasated red blood cells. It also shows (B) fibrinoid necrosis of the vessels wall (arrow). Findings are consistent with leukocytoclastic vasculitis.

\section{Management}

The patient was diagnosed with $\mathrm{CP}$ secondary to RV and was started on pulse methylprednisolone treatment. He was then transitioned to oral prednisone on discharge.

\section{Follow-up}

The patient remained symptomatic for several months despite medical management, including the addition of methotrexate and later sulfasalazine to his regimen. A repeated cardiac MRI revealed persistent pericardial inflammation. He was eventually referred to cardiothoracic surgery and underwent pericardiectomy. He tolerated the surgery well with an improvement in his clinical status.

\section{Discussion}

$\mathrm{CP}$ is a pericardial disease that occurs due to scarring and loss of elasticity of the pericardium. Inelastic pericardium does not expand and thus prevents the physiological decrease in intrathoracic pressure during inspiration to be transmitted to the chambers of the heart. This leads to elevated filling pressures of the right ventricle, which in turn shifts the interventricular septum towards the left ventricle (LV) impairing LV filling [1].

$\mathrm{CP}$ can occur after any pericardial process. The majority of cases are attributed to idiopathic $\mathrm{CP}$, viral illness, and previous cardiac surgery [2]. Other common causes include radiation therapy to the chest and postinfectious (tuberculous) pericarditis. Less common causes also include trauma, uremia, drugs, and malignancies. Rarely, CP may be a manifestation of vasculitis and connective tissue disorders.

$\mathrm{RV}$ is one of the most severe extra-articular complications of RA and is almost always seen in patients with long-standing and advanced RA [3]. In our case, the diagnosis of RV was made in the absence of longstanding RA. Skin is most frequently affected, with a prevalence of $90 \%$ among all patients with RV. This is followed by peripheral nervous system involvement, with various manifestations including sensory, motor, and mixed polyneuropathy. The involvement of cardiac tissue is the third most prevalent and is present in about 30\% of patients with RV [3]. Pericarditis is a common manifestation, but myocarditis, coronary arteritis, and aortitis can also occur.

History and physical examination are essential in the diagnosis of CP. EKG may show non-specific ST-T changes. Laboratory investigations may suggest an inflammatory process. Echocardiography is usually the initial imaging test. It assesses pericardial thickness, the motion of the pericardium and the myocardium, motion of the septal wall, ventricular interdependence, and cardiac chambers filling pressures. A combination of findings on echocardiograms can have up to $89 \%$ sensitivity and $95 \%$ specificity for the diagnosis of CP [4]. Cardiac MRI provides morphological assessment and hemodynamic characteristics in patients with CP. It also determines the extent of the pericardial inflammation and provides input to guide treatment [5]. CT scan can reveal the presence of pericardial calcification in $25 \%$ of patients, and aid in the pre-operative assessment [6].

Histopathological demonstration of vasculitis is the confirmatory diagnostic test. Findings of infiltration of the vessel wall by inflammatory cells and neutrophilic nuclear remnants along with accompanying fibrinoid necrosis of the vessel wall are present in most patients with RV [7].

Treatment options for $\mathrm{CP}$ include medical and surgical therapy. Medical therapy can be considered for specific etiologies like tuberculous pericarditis and in cases with transient pericarditis. Medical therapy is 
also used to control symptoms of heart failure (mainly diuretics) when surgery is not feasible. Otherwise, pericardiectomy is the mainstay treatment for early refractory CP and chronic CP. It has high perioperative morbidity and mortality, and thus it should be considered cautiously. $83 \%$ of patients who undergo pericardiectomy are free of clinical symptoms [8], with an overall survival rate of around $60 \%$ at 10 years [9].

Due to the paucity of randomized trials and rarity of occurrence of RV, there are no established guidelines regarding treatment. Patients usually receive high doses of corticosteroids and immunosuppressive agents. A large, single-center retrospective study reported that two-thirds of patients were treated with corticosteroids and one-third with cyclophosphamide. Other immunosuppressive agents were less commonly used. Biologic agents, including anti-TNF agents, rituximab, and anakinra were also used. Despite treatment, however, mortality rates remain high [10].

\section{Conclusions}

$\mathrm{CP}$ occurs due to scarring and loss of elasticity of the pericardium. It rarely occurs as a manifestation of vasculitis. RV is an uncommon complication of RA that usually occurs in males with a long-standing history of RA. Despite treatment, both CP and RV pose treatment challenges and have poor survival outcomes.

\section{Additional Information \\ Disclosures}

Human subjects: Consent was obtained or waived by all participants in this study. Conflicts of interest: In compliance with the ICMJE uniform disclosure form, all authors declare the following: Payment/services info: All authors have declared that no financial support was received from any organization for the submitted work. Financial relationships: All authors have declared that they have no financial relationships at present or within the previous three years with any organizations that might have an interest in the submitted work. Other relationships: All authors have declared that there are no other relationships or activities that could appear to have influenced the submitted work.

\section{References}

1. Sengupta PP, Eleid MF, Khandheria BK: Constrictive pericarditis. Circ J. 2008, 72:1555-62. 10.1253/circj.cj08-0654

2. Haley JH, Tajik AJ, Danielson GK, Schaff HV, Mulvagh SL, Oh JK: Transient constrictive pericarditis: causes and natural history. J Am Coll Cardiol. 2004, 43:271-5. 10.1016/j.jacc.2003.08.032

3. Kishore S, Maher L, Majithia V: Rheumatoid vasculitis: a diminishing yet devastating menace . Curr Rheumatol Rep. 2017, 19:39. 10.1007/s11926-017-0667-3

4. Sengupta PP, Mohan JC, Mehta V, Arora R, Pandian NG, Khandheria BK: Accuracy and pitfalls of early diastolic motion of the mitral annulus for diagnosing constrictive pericarditis by tissue Doppler imaging. Am J Cardiol. 2004, 93:886-90. 10.1016/j.amjcard.2003.12.029

5. Feng D, Glockner J, Kim K, et al.: Cardiac magnetic resonance imaging pericardial late gadolinium enhancement and elevated inflammatory markers can predict the reversibility of constrictive pericarditis after antiinflammatory medical therapy: a pilot study. Circulation. 2011, 124:1830-7. 10.1161/CIRCULATIONAHA.111.026070

6. Alajaji W, Xu B, Sripariwuth A, et al.: Noninvasive multimodality imaging for the diagnosis of constrictive pericarditis. Circ Cardiovasc Imaging. 2018, 11 :e007878. 10.1161/CIRCIMAGING.118.007878

7. Koutkia P, Mylonakis E, Rounds S, Erickson A: Leucocytoclastic vasculitis: an update for the clinician. Scand J Rheumatol. 2001, 30:315-22. 10.1080/030097401317148499

8. Ling LH, Oh JK, Schaff HV, Danielson GK, Mahoney DW, Seward JB, Tajik AJ: Constrictive pericarditis in the modern era: evolving clinical spectrum and impact on outcome after pericardiectomy. Circulation. 1999, 100:1380-6. 10.1161/01.cir.100.13.1380

9. DeValeria PA, Baumgartner WA, Casale AS, et al.: Current indications, risks, and outcome after pericardiectomy. Ann Thorac Surg. 1991, 52:219-24. 10.1016/0003-4975(91)91339-w

10. Makol A, Crowson CS, Wetter DA, Sokumbi O, Matteson EL, Warrington KJ: Vasculitis associated with rheumatoid arthritis: a case-control study. Rheumatology (Oxford). 2014, 53:890-9.

10.1093/rheumatology/ket475 